\title{
Distributional notes on some Nosodendridae (Coleoptera) - XXIII. Description of a new species from the Philippines
}

\section{Замечания о распространении видов семейства Nosodendridae (Coleoptera) - XXIII. Описание нового вида с Фимиппин}

\section{J. Háva \\ И. Гава}

Forestry and Game Management Research Institute, Strnady 136, Praha 5 -Zbraslav CZ-156 00 Czech Republic. E-mail: jh.dermestidae@volny.cz. ORCID ID: 0000-0001-8076-9538.

Научно-исследовательский институт охотничьего хозяйства, Стрнады 136, Praha 5 - Збраслав CZ-156 00 Чешская Республика.

Key words: taxonomy, description, new species, Coleoptera, Nosodendridae, Nosodendron, Philippines.

Ключевые слова: таксономия, описание, новый вид, Coleoptera, Mycetophagidae, Nosodendron, Филиппины.

Abstract. A new species Nosodendron (Dendrodipnis) skalickyi sp.n. from the Philippines is described, illustrated and compared with a similar species, Nosodendron (Dendrodipnis) barsevskisi Háva, 2019.

Pезюме. В статье описан новый вид Nosodendron (Dendrodipnis) skalickyi sp.n. с Филиппин, близкий к Nosodendron (Dendrodipnis) barsevskisi Háva, 2019.

\section{Introduction}

The small family Nosodendridae (Coleoptera) recently contains 2 genera and 103 species, eight species have been known from the Philippines [Háva, 2015, 2017, 2018, 2019, 2020a, b]. The family Nosodendridae was placed to Derodontoidea by Bouchard et al. [2011]. McKenna et al. [2019] placed the family Nosodendridae to Nosodendroidea.

A new species from the Philippines is described below.

\section{Material and methods}

Locality labels of the mentioned material are cited in the original version. Specimens of the presently described species are provided with red, printed labels with the text as follows: «HOLOTYPE (or PARATYPE, respectively) Nosodendron (Dendrodipnis) skalickyi sp. nov. Jiří Háva det. 2021».

Type material is deposited in collection:

JHAC - Jiří Háva, Private Entomological Laboratory and Collection, Prague-west, Czech Republic.

\section{Results}

Nosodendron (Dendrodipnis) skalickyi Háva, sp.n. Figs 1, 3, 5 .

Type material. Holotype: $\sigma^{7}-$ Philippines, Mindanao, Bukidnon, Dominorog, September 2019, local collector, (JHAC). Paratypes: $20^{7} \sigma^{7}$ - the same data as holotype, (JHAC).
Description. Male. Measurements (in $\mathrm{mm}$ ): total length 5.4; maximum elytral width 3.2. Body convex. Cuticle black, shiny. Head finely, but clearly punctate, with very short yellow setae. Eyes large, visible from above. Antennae brown, with eleven antennomeres, antennal club with three antennomeres, with yellow setae. Mentum with small punctures, with short yellow setae and with U shaped groove (Fig. 1). Pronotum punctate as head; with very short yellow setae. Pronotal anterior angle without groove. Scutellum triangular, finely punctate. Elytra with fine, short, yellow setae; each elytron with small punctures intermixed with small setabearing punctuation. Near scutellum each elytron with one short stria consisting of large punctures. Mesosternum finely and regularly punctate. Metasternum with longitudinal carina, punctures large on anterior margins, other punctures small. Legs black, tibiae and femora finely punctate, with short yellow setation. Abdominal visible ventrites black. Longitudinal striation of base of abdominal ventrites very short with punctures. Ventrite V (Fig. 3). Male genitalia (Fig. 5).

Female. Externally similar to male. Measurements (in $\mathrm{mm}$ ): total length 5.1-5.2; maximum elytral width 3.03.1

Comparision. The new species is very similar to Nosodendron (Dendrodipnis) barsevskisi Háva, 2019, but differs from it in the mentum, abdominal ventrite 5 and the male genitalia (Figs 1-6).

Etymology. Patronymic, dedicated to my friend and coleopterologist Stanislav Skalický (Ústí nad Orlicí, Czech Republic), specialist in Heteroceridae (Coleoptera).

\section{Check-list of Nosodendridae recorded from the Philippines}

\footnotetext{
Nosodendridae Erichson, 1846

Nosodendron Latreille, 1804

Nosodendron (Dendrodipnis) Wollaston, 1873

Nosodendron barsevskisi Háva, 2019 - Palawan; Mindanao

Nosodendron bolmi Háva, 2015 - Mindanao

Nosodendron hispidum Champion, 1923 - Palawan

Nosodendron lentum Oehme-Leonhardt, 1954 - Mindanao

Nosodendron luzonicus Háva, 2018 - Luzon
} 
Nosodendron mindanaoensis Háva, 2018 - Mindanao Nosodendron parahispidum Háva, 2015 - Mindanao Nosodendron planus Háva, 2015 - Mindanao Nosodendron skalickyi sp.n. — Mindanao

\section{Acknowledgements}

I am indebted very much to Jan Hrdlička (Czech Republic), for his help with the material from the Philippines. The paper was supported by the Ministry of Agriculture of the Czech Republic, institutional support MZE-RO0118.

\section{References}

Bouchard P., Bousquet Y., Davies A.E., Alonso-Zarazaga M.A., Lawrence J.F., Lyal Ch.H.C., Newton A.F., Reid Ch.A.M., Schmitt M., Ślipiński S.A., Smith A.B.T. 2011. Family-group names in Coleoptera (Insecta) // ZooKeys. Vol.88. P.1-972.

Háva J. 2015. Distributional notes on some Nosodendridae (Coleoptera) - XIV. Descriptions of three new species and new faunistics records from Philippines // Entomologische Zeitschrift. Vol.125. P.223-224.

Háva J. 2017. Distributional notes on some Nosodendridae (Coleoptera) - XVI. New faunistics records from the Philippines // Acta Biologicae Universitates Daugavpiliensis. Vol.17. No.1. P.5-7.

Háva J. 2018. Distributional notes on some Nosodendridae (Coleoptera) - XVIII. Descriptions of two new species from the Philippines // Folia Heyrovskyana. Series A. Vol.26. No.2. P.1-3.

Háva J. 2019. Distributional notes on some Nosodendridae (Coleoptera) - XX. Description of a new species from the Philippines // Euroasian Entomological Journal. Vol.18. No.6. P.409-411.

Háva J. 2020a. Distributional notes on some Nosodendridae (Insecta, Coleoptera) - XXI. A new Nosodendron species from Malaysia // Linzer Biologische Beiträge. Vol.52. No.1. P. 81-84.

Háva J. 2020b. Distributional notes on some Nosodendridae (Coleoptera) - XXII. A new species of Nosodendron (Nosodendron) from India // Natura Somygyiensis. Vol.35. P. 25-28.

McKenna D.D., Shin S., Ahrens D., Balke M., Beza-Beza C., Clarke D. J., Donath A., Escalona H.E., Friedrich F., Letsch H., Liu S., Maddison D., Mayer Ch., Misof B., Murin P.J.,
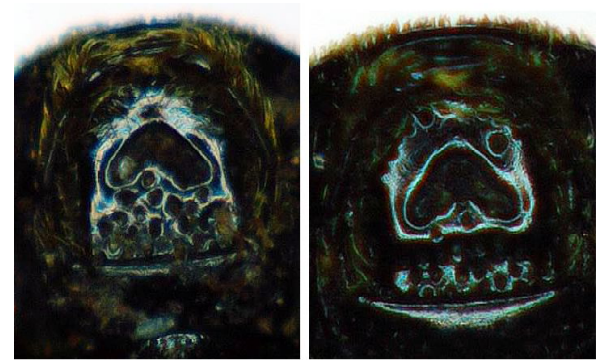

1
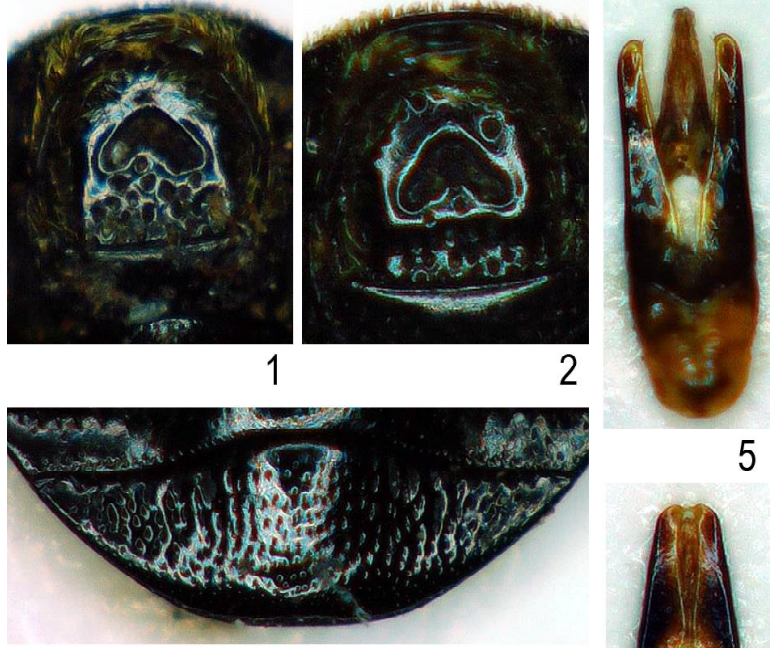

5

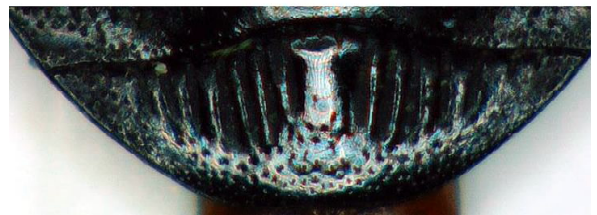

3

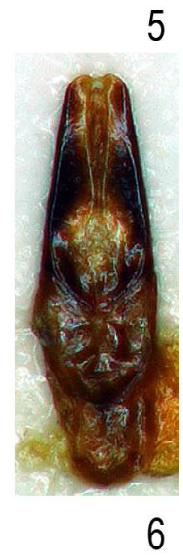

Figs 1-6. Nosodendron spp:: $1-$ N. skalickyi sp. nov., mentum; $2-N$. barsevskisi, mentum; $3-N$. skalickyi sp. nov., abdominal ventrite $5 ; 4-N$. barsevskisi, abdominal ventrite 5 5 - N. skalickyi sp. nov., male genitalia; $6-N$. barsevskisi, male genitalia.

Рис. 1-6. Nosodendron spp.: 1 - N. skalickyi sp. nov., подбородок; 2 - N. barsevskisi, подбородок; $3-N$. skalickyi sp. nov., 5-й вентрит; $4-$ N. barsevskisi, 5-й вентрит; $5-N$. skalickyi sp. nov., гениталии самца; $6-$ N. barsevskisi, гениталии самца.

Niehuis O., Peters R.S., Podsiadlowski L., Pohl H., Scully E.D., Yan E.V., Zhou X., Ślipiński A., Beutel R.G. 2019. The evolution and genomic basis of beetle diversity

// PNAS. Vol.116. No.49. P.24729-24737. 